\title{
A Simple Fight Decision Support System for BVR Air Combat Using Fuzzy Logic Algorithm
}

\author{
Khairul Ummah ${ }^{1,}{ }^{*}$, Herlan Setiadi ${ }^{1}$, Hisar Manongam Pasaribu' ${ }^{1}$, Dhani Anandito ${ }^{1}$ \\ 1 Bandung Institute of Technology, Bandung 40132, Indonesia \\ * Correspondence: khairulu@ae.itb.ac.id
}

Received: 10 August 2019; Accepted: 18 November 2019; Published: 30 December 2019

\begin{abstract}
Beyond Visual Range (BVR) air combat is a future trend of war tactic. In this situation, a fighter can attack the opponent before direct encounter. Its complexity arises due to the necessity to take into account the information of target's maneuver, the specification of the missile, and the advantage of fighter position. In this paper, a simple BVR air combat system has been developed to give a fight strategy for pilot. Some important parameters are considered, such as the distance and the azimuth position of the target's as well as the range and the energy of missile to reach the target. The information is processed to determine the fighter supremacy and the opponent's threat factor. The result of the processing is used as an input of fuzzy logic algorithm to determine the optimal fighting strategy. The feasibility of the model and validity of the algorithm are verified by simulation under two typical situations.
\end{abstract}

Keywords: beyond visual range, air combat, fuzzy logic, fighting strategy

\section{Introduction}

The development of air combat tactic using artificial intelligence is important in modern air combat. In [1]-[3] the air combat situations are analysed to support the pilot in making a strategic maneuver. Artificial intelligence is used to make the pilot's decision.

This study uses fuzzy logic algorithm to determine the optimal strategy for pilot. The recommendation strategy consists of all-out attack, fight defensively, and run away. In [4], an important opponent movement information is needed for analysis. The opponent movement can be categorized as rear approaching, rear flanking, rear escaping, sides approaching, sides flanking, sides escaping, front approaching, front flanking, front escaping. This information is crucial for the pilot to reduce risk encounter the target which less probability he can win.

\section{Methods}

This study uses fuzzy logic to calculate the Beyond Visual Range (BVR) decision making parameters. The input is the opponent's position (latitude, longitude and altitude), opponent's speed and direction of crossing flight (velocity and heading) and opponent's supremacy which is a function of aircraft type, sensor and armament capabilities.

The method consists of three main process, namely aircraft identification, target intension judgment, and supremacy calculation. The aircraft identification gathers and analyses RCS data, which is combined with weaponry database to calculate the threat factor. The target intension judgment is calculated by analysing radar data including location, velocity vector, and impact angle. 
The final step is calculating supremacy factor based on the threat and intension factor, as presented in Figure 1.

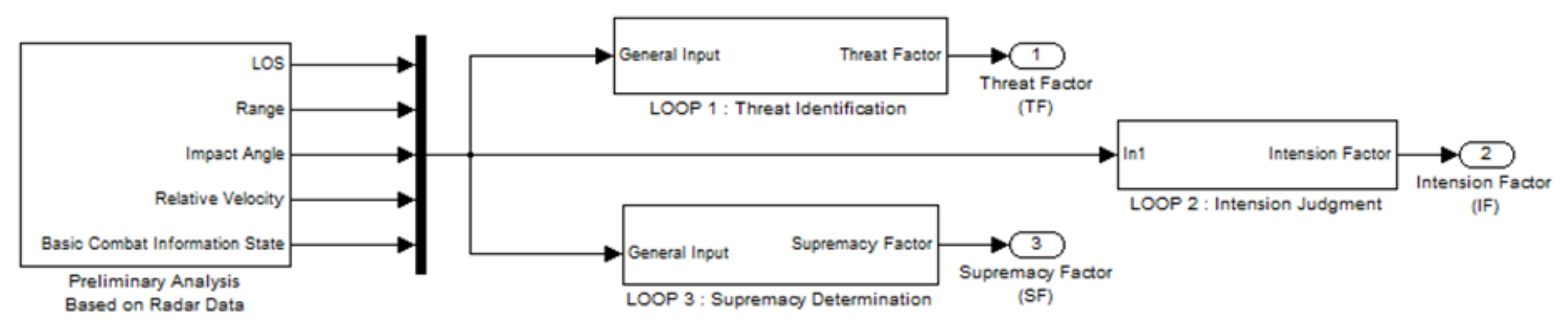

Figure 1. Fuzzy logic diagram of the target intention judgment

The supremacy factor is calculated by using supremacy function $\boldsymbol{S}$, as given below

$$
S=\alpha_{p}\left(\beta_{d} f_{d}+\beta_{\varphi} f_{\varphi}+\beta_{\theta} f_{\theta}\right)+\alpha_{E} f_{E}
$$

Where

$\alpha_{\mathrm{d}}$ is weight for the total position supremacy

$\alpha_{\mathrm{E}}$ is weight for the total energy supremacy

$\beta_{\mathrm{d}}$ is weight for the distance supremacy

$\beta_{\varphi}$ is weight for the azimuth supremacy

$\beta_{\ominus}$ is weight for the impact angle supremacy

$\mathrm{fd}_{\mathrm{d}}$ is function for the distance supremacy

$\mathrm{f}_{\varphi}$ is function for the azimuth supremacy

$f_{\ominus}$ is function for the impact angle supremacy

The same detail of the function can be found also in other literatures [2,3]. To make decision, the supremacy factor is combined with superiority factor. The Superiority factor depends on agility, flight performance, and weaponry of the aircraft. The detail of the superiority factor can be found in [4].

Supremacy and superiority factors have a role as the input of fuzzy logic algorithm to determine 3 decision, they are including:

1. All-out attack

2. Fight defensively

3. Run away

Rule as an input in fuzzy logic

1. if (supremacy factor is low and superiority factor is high) then fight defensively

2. if (supremacy factor is low and superiority factor is mid) then run away

3. if (supremacy factor is low and superiority factor is low) then run away

4. if (supremacy factor is medium and superiority factor is high) then all-out attack

5. if (supremacy factor is medium and superiority factor is medium) then fight defensively

6. if (supremacy factor is medium and superiority factor is low) then fight defensively

7. if (supremacy factor is high and superiority factor is high) then all-out attack

8. if (supremacy factor is high and superiority factor is medium) then all-out attack

9. if (supremacy factor is high and superiority factor is low) then all-out attack 


\section{Simulations}

Two simulations are performed using X-Plane simulator to test the method effectiveness. One is the direct encounter situation, named as 'approaching', and the other is chasing situation, named as 'escaping'.

\section{Approaching}

In this simulation, the attacker $>$ and the target $>$ are approaching each other. The attacker has the advantage of higher altitude position than the target. It also has a higher (medium) superiority, as presented in Figure 2.

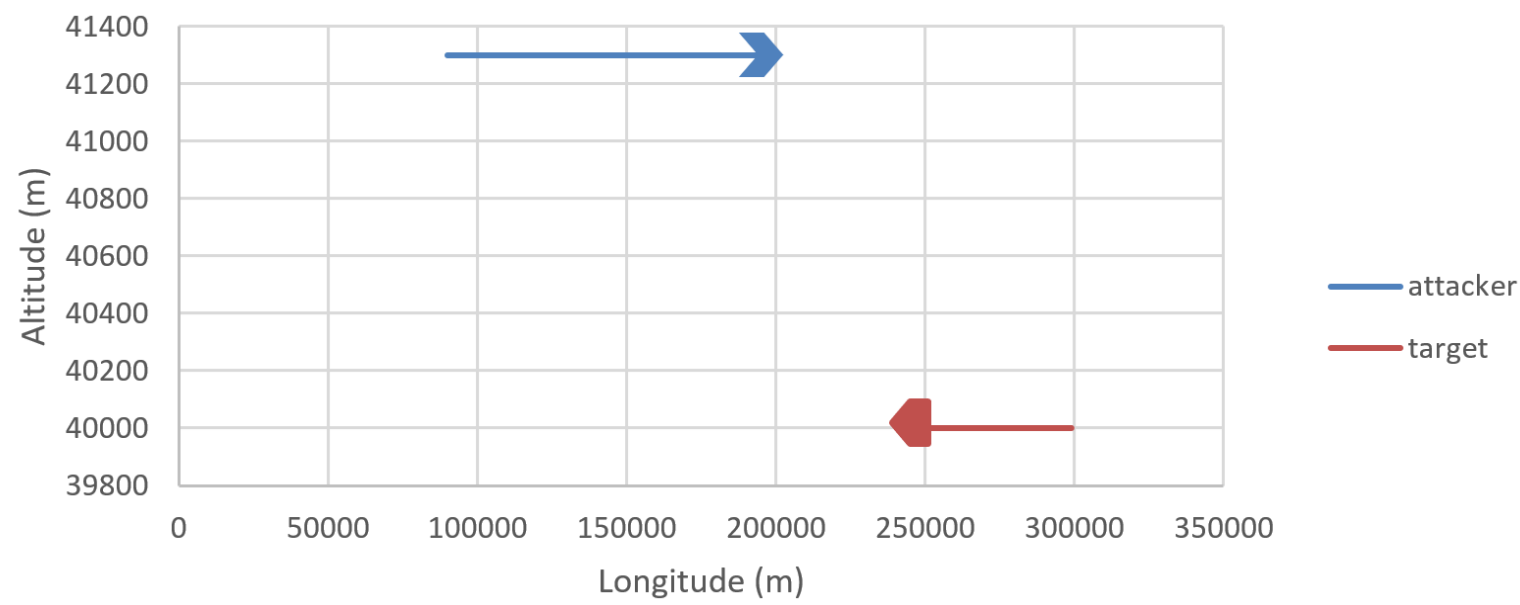

Figure 2. The attacker and the target approach each other

In Figure 3, the supremacy factor is increasing along with decreasing distance. It is because the target approach to the attacker's radar and missile detection area.

The decision strategy from fuzzy logic is shifting from fight defensively into all-out attack when the supremacy factor around 0.69 (from rule 5 to rule 8). In this case, the value of superiority factor is constant, but the supremacy factor is increased. This situation suggests the attacker to the attack target as presented in Figure 3.

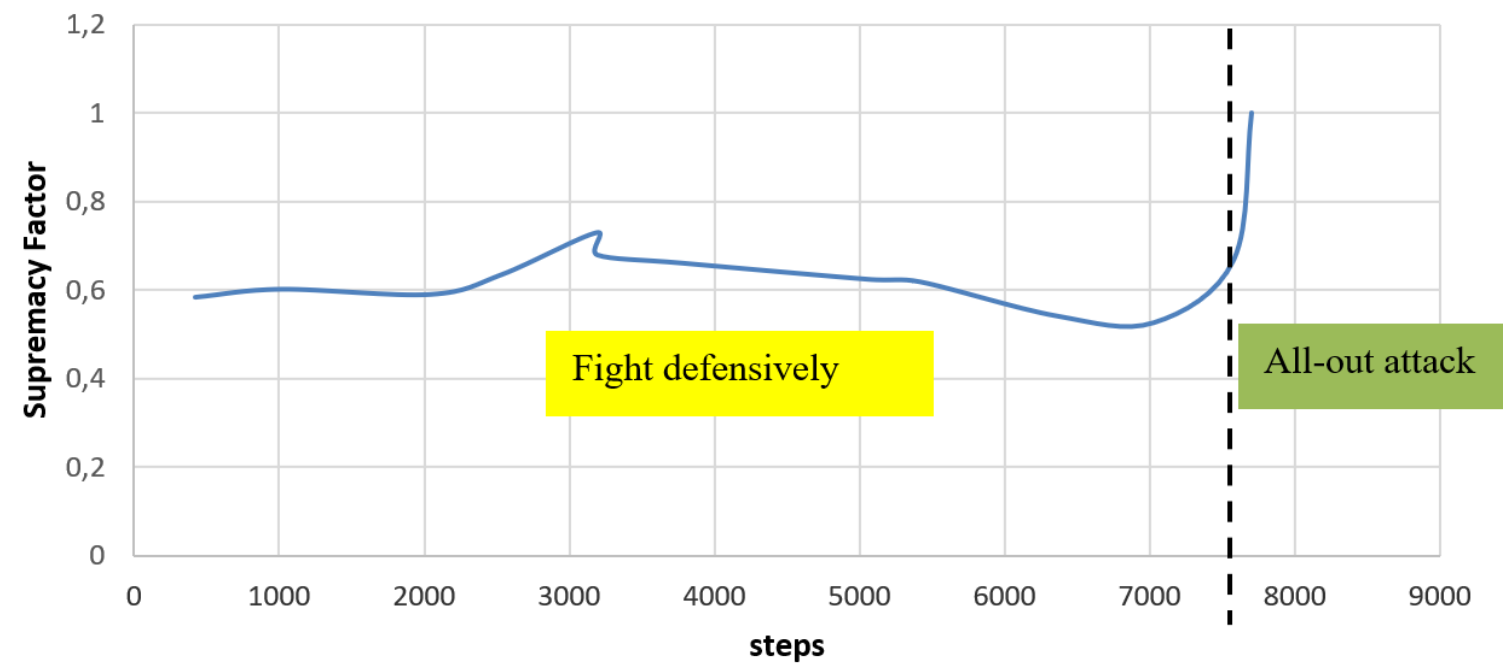

Figure 3. Supremacy factor for simulation when the attacker and the target approach each other 


\section{Escaping}

In this simulation the attacker

Figure 4. But the target can escape with higher velocity. The attacker has a higher (medium) superiority than the target.

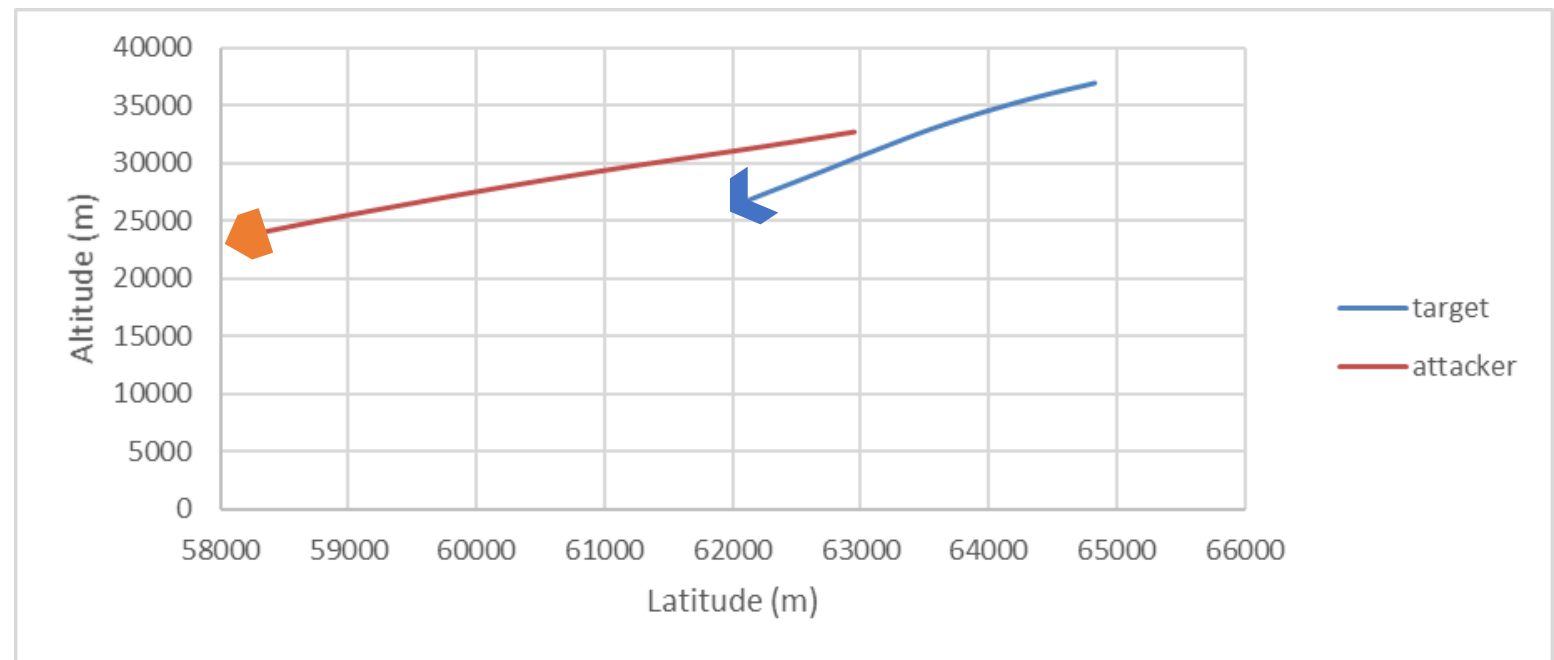

Figure 4. The attacker pursuit the target, but the target can escape with higher velocity

In Figure 5, the supremacy factor is decreasing along with increasing distance. It is because the target escape from the attacker's radar and missile detection area. The decision strategy from fuzzy logic is shifting from all-out attack into fight defensively when the supremacy factor around 0.7 (from rule 8 to rule 5). In this case, the value of superiority factor is constant but the supremacy factor is decreased to change the applied rule in fuzzy logic.

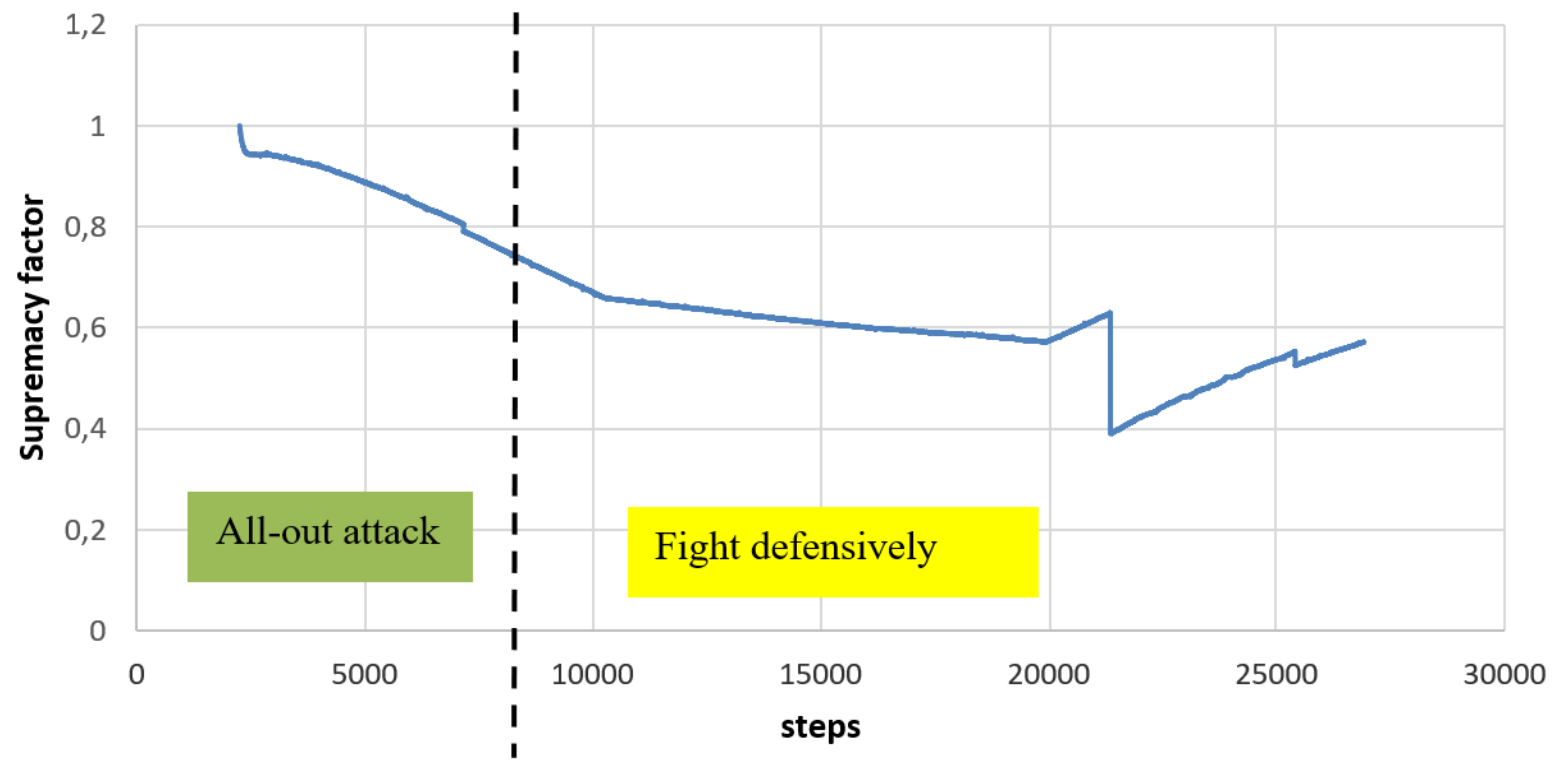

Figure 5. Supremacy factor for simulation when the target escape from the attacker 


\section{Conclusions}

In this paper, automatic fight mode decision strategy in BVR air combat by using fuzzy logic algorithm was presented. Moreover, our method to automate strategy recommendation for BVR air combat has been proposed. The validity and verified information about the target are very important as an input in this method. In future work, the method will be extended to give recommendation for the maneuver strategy.

\section{References}

1. James S, Jonathan P, Brian W, et al, 2010. Air combat strategy using approximate dynamic programming Journal of Guidance, Control and Dynamic 33 1641-1654

2. Xiao, L. and Huang, J., 2011. Maneuver Strategy in Beyond-Visual-Range Air Combat. Proceedings of the 2011 International Conference on Information Communication and Management, Singapore, October 14-15.

3. Tao Zhang, Lei Yu and Zhongliang Zhou, 2012. Decision making for Air Combat Maneuvering Based on Variable Weight Pseudoparallel Genetic Algorithm Flight Dynamics 30 470-474

4. Anandhito, D., 2016. Analisis Superiority Factor Armada Pesawat Tempur untuk Studi Combat Effectiveness. Undergraduate's thesis, Institut Teknologi Bandung, Faculty of Mechanical and Aerospace Engineering.

(C) 2019 by the authors. Submitted for possible open access publication under the terms and conditions of the Creative Commons Attribution (CC BY) license (http://creativecommons.org/licenses/by/4.0/). 